\title{
Superior CKIP-1 sensitivity of orofacial bone-derived mesenchymal stem cells in proliferation and osteogenic differentiation compared to long bone-derived mesenchymal stem cells
}

\author{
XIN HUANG ${ }^{1,2}$, BINGKUN CHENG $^{2}$, WEN SONG $^{3}$, LE WANG $^{2}$, YANYUAN ZHANG $^{2}$, \\ YAN $\mathrm{HOU}^{2}, \mathrm{YU} \mathrm{SONG}^{4}$ and LIANG KONG ${ }^{2}$ \\ ${ }^{1}$ School of Stomatology of Qingdao University, Qingdao, Shandong 266003; \\ ${ }^{2}$ State Key Laboratory of Military Stomatology and National Clinical Research Center for Oral Diseases \\ and Shaanxi Clinical Research Center for Oral Diseases, Department of Oral and Maxillofacial Surgery, \\ School of Stomatology, The Fourth Military Medical University; ${ }^{3}$ State Key Laboratory of Military Stomatology \\ and National Clinical Research Center for Oral Diseases and Shaanxi Key Laboratory of Stomatology, \\ Department of Prosthodontics, School of Stomatology, The Fourth Military Medical University, \\ Xi'an, Shaanxi 710032; ${ }^{4}$ Department of Orthodontics, Qingdao Stomatological Hospital, \\ Qingdao, Shandong 266001, P.R. China
}

Received October 14, 2019; Accepted April 9, 2020

DOI: $10.3892 / \mathrm{mmr} .2020 .11239$

\begin{abstract}
Maxillofacial bone defects caused by multiple factors, including congenital deformations and tumors, have become a research focus in the field of oral medicine. Bone tissue engineering is increasingly regarded as a potential approach for maxillofacial bone repair. Mesenchymal stem cells (MSCs) with different origins display various biological characteristics. The aim of the present study was to investigate the effects
\end{abstract}

Correspondence to: Professor $\mathrm{Yu}$ Song, Department of Orthodontics, Qingdao Stomatological Hospital, 17 Dexian Road, Qingdao, Shandong 266001, P.R. China

E-mail: songyusy888@163.com

Professor Liang Kong, State Key Laboratory of Military Stomatology and National Clinical Research Center for Oral Diseases and Shaanxi Clinical Research Center for Oral Diseases, Department of Oral and Maxillofacial Surgery, School of Stomatology, The Fourth Military Medical University, 145 West Changle Road, Xi'an, Shaanxi 710032, P.R. China

E-mail: kongliang@fmmu.edu.cn

Abbreviations: CKIP-1, casein kinase-2 interaction protein-1; BMSCs, mesenchymal stem cells; OMSCs, orofacial bone-derived mesenchymal stem cells; BMMSCs, long bone marrow-derived mesenchymal stem cells; WT, wild-type; KO, knockout; CKIP-1 ${ }^{-/}$, CKIP-1 knockout; Smurf1, Smad ubiquitination regulatory factor 1; HA/ $\beta$-TCP, hydroxyapatite/tricalcium phosphate

Key words: CKIP-1, OMSCs, BMMSCs, osteogenic differentiation, ectopic bone formation of casein kinase-2 interaction protein-1 (CKIP-1) on MSCs, including femoral bone marrow-derived MSCs (BMMSCs) and orofacial bone-derived MSCs (OMSCs), isolated from the femoral and orofacial bones of wild-type (WT) and CKIP-1 knockout (KO) mice. MSCs were isolated using collagenase II and the main biological characteristics, including proliferation, apoptosis and osteogenic differentiation, were investigated. Subcutaneous transplantation of MSCs in mice was also performed to assess ectopic bone formation. MTT and clone formation assay results indicated that cell proliferation in the KO group was increased compared with the WT group, and OMSCs exhibited significantly increased levels of proliferation compared with BMMSCs. However, the proportion of apoptotic cells was not significantly different between CKIP-1 KO OMSCs and BMMSCs. Furthermore, it was revealed that osteogenic differentiation was increased in CKIP-1 KO MSCs compared with WT MSCs, particularly in OMSCs. Consistent with the in vitro results, enhanced ectopic bone formation was observed in CKIP-1 KO mice compared with WT mice, particularly in OMSCs compared with BMMSCs. In conclusion, the present results indicated that OMSCs may have a superior sensitivity to CKIP-1 in promoting osteogenesis compared with BMMSCs; therefore, CKIP-1 KO in OMSCs may serve as an efficient strategy for maxillofacial bone repair.

\section{Introduction}

Maxillofacial bone defects are a problem in oral medicine and are caused by congenital deformations, traumatic fractures, infections or tumors (1). Bone tissue engineering is considered as a primary option for bone defect repair $(2,3)$. Therefore, mesenchymal stem cells (MSCs) are in high demand for the 
regenerative restoration of maxillofacial bone defects (4). The osteogenic capacity of MSCs can be affected by a number of factors, including long noncoding RNAs (5), microRNAs (6), circular RNAs (7), chemical drugs (8) and topographically optimized scaffolds (9). Moreover, the osteogenic capacity of MSCs is closely related to their tissue of origin (4,10-13). For example, it has been reported that the osteogenic differentiation ability of MSCs derived from cartilage (12) and adipose tissue $(12,13)$ are decreased compared with bone marrow-derived MSCs (BMMSCs). Furthermore, MSCs tend to differentiate into the tissue from which they originate (14); therefore, orofacial MSCs (OMSCs) are the optimal choice for maxillofacial bone defect restoration. However, the osteogenic characteristics of OMSCs have not been fully elucidated, particularly in comparison with BMMSCs, which are commonly isolated from the bone marrow of the femur (15). Considering that the mandible and femur have different modes of bone formation during embryonic development (16), it was hypothesized that the osteogenic differentiation ability of OMSCs and BMMSCs may differ.

Casein kinase-2 interaction protein-1 (CKIP-1) negatively regulates bone formation via Smad ubiquitination regulatory factor 1 (Smurf1) signaling (17). Our previous study also reported that CKIP-1 silencing promoted new bone formation during mandibular distraction osteogenesis in rats (18). Moreover, in CKIP- $1^{-/}$mice, the level of mandible formation was significantly increased compared with femur bone formation (unpublished data), which further indicated that cellular responses to CKIP-1 knockout (KO) may be significantly different.

In the present study, OMSCs and BMMSCs were isolated from wild-type (WT) and knockout (KO; CKIP-1 $\left.{ }^{-/}\right)$mice. Furthermore, the effect of CKIP-1 KO on the proliferation and osteogenic differentiation of OMSCs and BMMSCs were investigated.

\section{Materials and methods}

Animals. In total, ten male C57BL/6 mice (age, 4 weeks; weight, $15 \pm 2 \mathrm{~g}$; obtained from the Animal Center of The Fourth Military Medical University), ten male CKIP-1 ${ }^{-/-}$mice (age, 4 weeks; obtained from the Institute of Radiation Medicine, Academy of Military Medical Sciences) and 40 male nude mice (age, 6 weeks; obtained from the Animal Center of The Fourth Military Medical University) were included in the study. A total of 10 nude mice were in each group: BMMSCs WT, BMMSCs KO, OMSCs WT and OMSCs KO. Mice were housed under light-, temperature-, and humidity-controlled conditions (12-h light/dark regimen; $22^{\circ} \mathrm{C}$; at a constant humidity of $55 \pm 5 \%$ ) with free access to food and water. All procedures in this study were approved by the Institutional Animal Care and Use Committee of The Fourth Military Medical University.

Genotype identification of CKIP- $1^{-/}$mice. Sections of the tails of CKIP-1 ${ }^{-/}$mice were collected in $1.5 \mathrm{ml}$ Eppendorf tubes and $200 \mu \mathrm{l}$ lysate prepared by $1 \mathrm{M}$ Tris ( $\mathrm{pH}=8.0$; Sangon Biotech Co., Ltd.), $2 \mathrm{M} \mathrm{NaCl}$ (Sangon Biotech Co., Ltd.), 0.5 M EDTA (Sangon Biotech Co., Ltd.), 10\% SDS (Sangon Biotech Co., Ltd.) and $\mathrm{ddH}_{2} \mathrm{O}$ was added. After incubation at $55^{\circ} \mathrm{C}$ for $20 \mathrm{~min}$, proteinase $\mathrm{K}$ (Takara Bio, Inc.) was inactivated by incubation at $95^{\circ} \mathrm{C}$ for $5 \mathrm{~min}$. The pyrolysis products were mixed by vortex oscillation, centrifuged at $12,000 \mathrm{x}$ g for $10 \mathrm{~min}$ at $4^{\circ} \mathrm{C}$ and the supernatant was used for PCR. The following primer pairs were used to identify the WT mice: CKIP-1WTN (+) forward, 5'-TGGTTTCCCCTCGGACCTGTAGGAAG-3'; CKIP-1500 reverse, 5'-TTCCCCCTTTGTGAAGCCCCAACTCTTGA CTC-3'. The following primer pairs were used to identify the CKIP-1 KO mice: CKIP-1WTN (+) forward, 5'-TGGTTTCCC CTCGGACCTGTAGGAAG-3'; Rin-1A+12bp (-) reverse, 5'-CCAGACTGCCTTGGGAAAAGCGCCTCC CCTACC-3'. Golden Star T6 Super PCR Mix kit (TsingKe Biotech Co., Ltd.) was used for PCR according to the manufacturer's protocol. The following thermocycling conditions were used: Initial denaturation step at $98^{\circ} \mathrm{C}$ for $2 \mathrm{~min}$; followed by 35 cycles at $98^{\circ} \mathrm{C}$ for $10 \mathrm{sec}$ and $58^{\circ} \mathrm{C}$ for $30 \mathrm{sec}$; and a final extension step at $72^{\circ} \mathrm{C}$ for $10 \mathrm{sec}$. Samples $(10 \mu \mathrm{l})$ and DL-2000 DNA Marker $(10 \mu \mathrm{l}$; Vazyme Biotech Co., Ltd.) were loaded in each chamber. Agarose gel electrophoresis (Biowest) on a 2\% gel was conducted under $180 \mathrm{~V}$ for $30 \mathrm{~min}$, and images were obtained under ultraviolet light.

Micro-computed tomography $(C T)$ scanning. CKIP-1 ${ }^{-/}$and WT mice were sacrificed by excessive sodium pentobarbital (150 mg/kg; Sigma-Aldrich; Merck KGaA), and subsequently the femurs were isolated and fixed with $4 \%$ paraformaldehyde (PFA; FeiYang Biotech Co., Ltd.) for $24 \mathrm{~h}$ at room temperature. Micro-CT (YXLON Cheetah; YXLON International $\mathrm{GmbH} ; 90 \mathrm{kV}, 45 \mu \mathrm{A}, 1,000 \mathrm{msec}$ ) was performed to scan the fixed femora with a layer thickness of $8 \mu \mathrm{m}$. Cancellous bone at the distal end of the femur and cortical bone in the middle part of the femur were selected as the area of interest for three-dimensional (3D) reconstruction. Bone volume/total volume ratio (BV/TV), trabecular number (Tb.N), trabecular thickness (Tb.Th), bone surface/bone volume (BS/BV), trabecular separation (Tb.Sp), cortical area (Ct.Ar), cortical inner diameter perimeter (Ct.ld.Pm), cortical outer diameter perimeter (Ct.Od.Pm), cortical thickness (Ct.Th), cortical bone volume (Ct.BV), trabecular area (Tb.Ar) and trabecular width (Tb.Wi) were selected as measurement indices, and quantified by Inveon ${ }^{\mathrm{TM}}$ Research Workplace 2.2 (Siemens AG).

Hematoxylin and eosin $(H \& E)$ staining. The samples were fixed with $4 \%$ PFA for $48 \mathrm{~h}$ at room temperature. After decalcification, paraffin embedding and deparaffinization, the samples were cut into 3-4 $\mu \mathrm{m}$ thick sections (Leica Microsystems $\mathrm{GmbH}$ ). Paraffin sections were stained with hematoxylin (Sigma-Aldrich; Merck KGaA) for $15 \mathrm{~min}$ at room temperature and washed three times with distilled water for $1 \mathrm{~min}$ each. After differentiation with $1 \%(\mathrm{v} / \mathrm{v})$ hydrochloric acid alcohol and washing with distilled water three times, hematoxylin-stained sections were stained with $0.5 \%$ eosin (Sigma-Aldrich; Merck $\mathrm{KGaA}$ ) for $3 \mathrm{~min}$ at room temperature and subsequently washed. Stained sections were observed using a DMI6000 inverted light microscope (Leica Microsystems $\mathrm{GmbH}$ ) at a magnification of x40.

Isolation and culture of BMMSCs and OMSCs. C57BL/6 mice (age, 4 weeks) were sacrificed by an overdose of sodium pentobarbital (150 mg/kg; Sigma-Aldrich; Merck KGaA), and 
subsequently the femora and mandibles were collected. The bones were cut into pieces and transferred to $25-\mathrm{cm}^{2}$ culture bottles (Costar; Corning, Inc.) containing $3 \mathrm{ml} \mathrm{0.25 \%} \mathrm{type} \mathrm{II}$ collagenase (Sigma-Aldrich; Merck KGaA) (19-21). After digestion on a swing bed at room temperature for $90 \mathrm{~min}$, collagenase activity was terminated using 10\% FBS (Gibco; Thermo Fisher Scientific, Inc.). Then, bone pieces were washed with $\alpha$-MEM (Gibco; Thermo Fisher Scientific, Inc.) supplemented with $2 \% \mathrm{FBS}$ and cultured at $37^{\circ} \mathrm{C}$ with $5 \% \mathrm{CO}_{2}$. Cell culture medium ( $\alpha$-MEM supplemented with $10 \%$ FBS and $1 \%$ streptomycin/penicillin) was replaced every 2 days, and the cells were passaged at $\sim 80 \%$ confluency. Cells at passages 3-5 were used for further analysis. After a fixation with $4 \%$ PFA for $24 \mathrm{~h}$ at room temperature and gold sputtering (22), bone pieces before and after digestion were observed by field emission scanning electron microscopy (FE-SEM; S-4800; Hitachi Co., Ltd). Cells of different generations were observed using an inverted phase contrast microscope (Nikon Corporation) at a magnification of $\mathrm{x} 100$.

Surface markers validation. Following digestion with $0.25 \%$ trypsin (Sigma-Aldrich; Merck KGaA), MSCs $\left(5 \times 10^{6}\right.$ cells $\left./ \mathrm{ml}\right)$ were seeded into Eppendorf tubes $(100 \mu \mathrm{l}$ per tube). Non-specific detection of the Fc component of the CD antibodies were blocked by $5 \%$ BSA (Sangon Biotech Co., Ltd.) for $30 \mathrm{~min}$ at room temperature. Anti-CD44 (1:100; cat. no. 553133; BD Biosciences), anti-CD29 (1:100; cat. no. 558741; BD Biosciences), anti-CD31 (1:100; cat. no. FAB3628P; R\&D Systems, Inc.), anti-CD34 (1:100; cat. no. 128609; BioLegend, Inc.) and anti-CD90 (1:100; cat. no. 105307; BioLegend, Inc.) antibodies were added and incubated at $37^{\circ} \mathrm{C}$ for $45 \mathrm{~min}$. Cells were washed twice with PBS, resuspended and subsequently analyzed by flow cytometry (FCM) using a BD FACSCanto ${ }^{\mathrm{TM}}$ II flow cytometer (BD Biosciences) and BD FACSDiva $^{\mathrm{TM}} 6.0$ software (BD Biosciences). Data analysis was performed using FlowJo software (version 7.2; FlowJo LLC) with a previously described gating method (23).

Multilineage differentiation. At $80 \%$ confluency, osteogenic and adipogenic differentiation were performed in osteogenic medium (growth medium containing $10 \mathrm{nM}$ dexamethasone, $10 \mathrm{mM} \beta$-glycerophosphate and $50 \mu \mathrm{g} / \mathrm{ml}$ ascorbic acid; all purchased from Sigma-Aldrich; Merck KGaA), and adipogenic medium ( $1 \mu \mathrm{mol} / 1$ dexamethasone, $10 \mathrm{mg} / 1$ insulin, $0.5 \mathrm{mmol} / 1$ IBMX and $100 \mu \mathrm{mol} / 1$ indometacin; all purchased from Sigma-Aldrich; Merck KGaA), respectively. After 21 days of osteogenic induction and 14 days of adipogenic induction at $37^{\circ} \mathrm{C}$, Alizarin Red S staining (ScienCell Research Laboratories, Inc.) and Oil Red O staining (Amresco, LLC) were performed to investigate the multidirectional differentiation potential of the cells, according to the manufacturer's instructions. Images were obtained using an inverted light microscope (Leica Microsystems $\mathrm{GmbH}$ ) at a magnification of $x 40$.

Cell morphology. Cells $\left(2 \times 10^{4}\right.$ cells $\left./ \mathrm{ml}\right)$ of the BMMSC WT, BMMSC KO, OMSC WT and OMSC KO groups were cultured on a coverslip for 1 day at $37^{\circ} \mathrm{C}$ prior to observation. Cells were rinsed with PBS and fixed with $3 \%$ glutaraldehyde overnight at room temperature. Subsequently, the cells were dehydrated with an ascending ethanol series (50, 70, 80, 90 and $100 \%$ ) and dried at room temperature. After gold sputtering, cell morphology was observed by FE-SEM using the aforementioned method.

Cell proliferation. Proliferation of MSCs was assessed using an MTT assay (Amresco, LLC). In total, $200 \mu \mathrm{l} \mathrm{MSC}$ suspension was seeded $\left(1 \times 10^{4}\right.$ cells/well) in 96-well plates and cultured at $37^{\circ} \mathrm{C}$. Subsequently, $20 \mu \mathrm{l}$ MTT was added to each well for $4 \mathrm{~h}$ at $37^{\circ} \mathrm{C}$. The medium was removed and $150 \mu \mathrm{l}$ DMSO was added to each well. The absorbance was measured at a wavelength of $490 \mathrm{~nm}$ using a microplate reader (Omega Bio-Tek, Inc.).

Clone formation assay. Cells were seeded ( $2.5 \times 10^{3}$ cells $)$ into a $10-\mathrm{cm}$ dish and routinely cultured for $\sim 14$ days at $37^{\circ} \mathrm{C}$. The clones were rinsed with PBS and fixed with 4\% PFA for $30 \mathrm{~min}$ at room temperature. Subsequently, the clones were stained with $1 \%$ toluidine blue for $30 \mathrm{~min}$ at room temperature and visualized using an inverted light microscope (Leica Microsystems $\mathrm{GmbH}$ ) at a magnification of $\mathrm{x} 100$. Clones containing $\geq 50$ cells were counted using Image-Pro Plus software (version 7.1; Media Cybernetics, Inc.), and the clone formation rate was calculated using the following equation: (The number of clone colonies/number of seeded cells) x100\%.

Cell apoptosis. Apoptotic BMMSCs and OMSCs in the $\alpha$-MEM culture medium (Gibco; Thermo Fisher Scientific, Inc.) and adherent cells were collected and washed with PBS. Cells were adjusted to a density of $1 \times 10^{6} / \mathrm{ml}$ and $1 \mathrm{ml}$ cell suspension was centrifuged at $1,500 \mathrm{x}$ g for $5 \mathrm{~min}$ at room temperature. Subsequently, the cells were resuspended in $500 \mu \mathrm{l}$ binding buffer (Biomiga, Inc.). Apoptotic cells were stained with $5 \mu \mathrm{l}$ Annexin V-FITC and $10 \mu \mathrm{l}$ propidium iodide for $10-15 \mathrm{~min}$ at room temperature, and analyzed by FACSCanto II (BD Biosciences). Cells from the lower left, the lower right, the upper right and the upper left represent normal, early, late and dead cells, respectively. The apoptotic rate was calculated as the sum of early and late apoptosis.

Osteogenic capacity evaluation. Cells of the BMMSC WT, BMMSC KO, OMSC WT and OMSC KO groups were seeded $\left(2 \times 10^{5}\right.$ cells $\left./ \mathrm{ml}\right)$ into 6 -well plates and cultured for 1 day at $37^{\circ} \mathrm{C}$. Subsequently, the $\alpha$-MEM culture medium (Gibco; Thermo Fisher Scientific, Inc.) was replaced with osteogenic inducing fluid prepared using the aforementioned method, which was replaced every 2 days. Following 7 and 21 days of osteogenic induction at $37^{\circ} \mathrm{C}$, cells were fixed with $4 \%$ PFA for $30 \mathrm{~min}$ at room temperature. Alkaline phosphatase (ALP) staining (LeaGene Biotech Co., Ltd.) and Alizarin Red staining (ScienCell Research Laboratories, Inc.) were performed to assess osteogenic differentiation according to the manufacturer's protocol. Differentiated cells were observed using an inverted light microscope (Leica Microsystems $\mathrm{GmbH}$ ) at a magnification of $\mathrm{x} 40$.

Reverse transcription-quantitative PCR (RT-qPCR). RT-qPCR was performed to assess the mRNA expression levels of CKIP-1 and osteogenesis-associated genes, including the early-expressed genes RUNX family transcription factor 2 (Runx2) and ALP, 
and the late-expressed genes colicinogenic factor 1 (COL1) and bone $\gamma$-carboxyglutamate protein (OCN). Total RNA was extracted from the induced cells using TRIzol ${ }^{\circledR}$ reagent (Invitrogen; Thermo Fisher Scientific, Inc.) according to the manufacturer's protocol. Reverse transcription was performed using PrimeScript RT Master Mix (Takara Bio, Inc.) in a 20- $\mu 1$ volume. The following temperature protocol was used for reverse transcription: $37^{\circ} \mathrm{C}$ for $15 \mathrm{~min}, 85^{\circ} \mathrm{C}$ for $5 \mathrm{sec}$ and $4^{\circ} \mathrm{C}$ for 10 min. qPCR was performed using SYBR PCR Master Mix kit (Takara Bio, Inc.), $10 \mu \mathrm{M}$ specific primers in a $25-\mu \mathrm{l}$ volume and an iCycleri QTX detection system (Bio-Rad Laboratories, Inc.). The following thermocycling conditions were used: Initial denaturation step at $95^{\circ} \mathrm{C}$ for $1 \mathrm{~min}$; followed by 35 cycles at $95^{\circ} \mathrm{C}$ for $30 \mathrm{sec}, 58^{\circ} \mathrm{C}$ for $30 \mathrm{sec}$; and a final extension step at $72^{\circ} \mathrm{C}$ for $30 \mathrm{sec}$. All signals were normalized to $\beta$-actin, and the $2^{-\Delta \Delta \mathrm{Cq}}$ method was used for quantification (24). The primers used for RT-qPCR are presented in Table I.

Ectopic bone formation. Hydroxyapatite (HA) and tricalcium phosphate $(\beta-\mathrm{TCP})$ scaffolds were provided by the National Engineering Research Center for Biomaterials. The scaffolds (40 wt.\% $\beta$-TCP and $60 \mathrm{wt} . \%$ HA; porosity, $60 \%$; pore size, $300-500 \mu \mathrm{m}$ ) were fabricated by sintering for $3 \mathrm{~h}$ at $1,100^{\circ} \mathrm{C}$. WT and CKIP- $1^{-/}$MSCs were seeded $\left(2 \times 10^{6}\right.$ cells $\left./ \mathrm{ml}\right)$ onto the surface of the HA/ $\beta$-TCP scaffolds and incubated for $30 \mathrm{~min}$ at room temperature with shaking. Subsequently, the scaffolds were incubated at $37^{\circ} \mathrm{C}$ with $5 \% \mathrm{CO}_{2}$ for $24 \mathrm{~h}$. The $\alpha$-MEM culture medium (Gibco; Thermo Fisher Scientific, Inc.) was replaced with osteogenic medium and incubated for 7 days at $37^{\circ} \mathrm{C}$, and the osteogenic medium was replaced every 2 days. The nude mice were anaesthetized by intraperitoneal injection of sodium pentobarbital (60 mg/kg; Sigma-Aldrich; Merck KGaA) and the prepared HA/ $\beta-T C P / M S C s$ scaffolds were subcutaneously transplanted into the anterior and posterior regions of the backs of nude mice. Mice were sacrificed after 2 months by an overdose of sodium pentobarbital $(150 \mathrm{mg} / \mathrm{kg}$; Sigma-Aldrich; Merck KGaA) and the scaffolds were isolated. Then, H\&E and Masson-trichrome staining were performed to assess osteogenesis. H\&E staining was carried out using the aforementioned method. For Masson-trichrome staining (Beijing Solarbio Science \& Technology Co., Ltd.), the samples were fixed with $4 \%$ PFA for $48 \mathrm{~h}$ at room temperature, and other steps were similar to the aforementioned H\&E staining. The staining was performed according to the manufacturer's protocols. After staining with hematoxylin for $10 \mathrm{~min}$, differentiated with $1 \%(\mathrm{v} / \mathrm{v})$ hydrochloric acid alcohol for $10 \mathrm{sec}$ and washed with distilled water three times at room temperature, the samples were incubated with Masson blue stain for $5 \mathrm{~min}$ at room temperature and then washed. Subsequently, acid fuchsin was added for $5 \mathrm{~min}$ at room temperature, and then samples were washed. After staining with $1 \%$ phosphomolybdic acid for $3 \mathrm{~min}$ at room temperature, aniline blue dye was finally added for $5 \mathrm{~min}$ and washed quickly with distilled water. Stained sections with a thickness of $\sim 3 \mu \mathrm{m}$ were observed using a DMI6000 inverted light microscope (Leica Microsystems $\mathrm{GmbH}$ ) at a magnification of $\mathrm{x} 40$. Image Pro Plus software (version 7.1; Media Cybernetics, Inc.) was used to analyze the percentage of new bone formation.

Statistical analysis. Statistical analyses were performed using SPSS software (version 19.0; SPSS, Inc.). Data are presented
Table I. Primers used for reverse transcription-quantitative PCR.

\begin{tabular}{ll} 
Gene & \multicolumn{1}{c}{ Primer sequence $\left(5^{\prime} \rightarrow 3^{\prime}\right)$} \\
\hline$\beta$-actin & F: CTGGCACCACACCTTCTAC \\
& R: GGTACGACCAGAGGCATAC \\
CKIP-1 & F: AACCGCTATGTGGTGCTGA \\
& R: CAGGGTGAACTTGCTGTGA \\
Runx2 & F: GGCCAGGTTCAACGATCTG \\
& R: GGACCGTCCACTGTCACTT \\
ALP & F: AACCTGACTGACCCTTCCC \\
& R: TTCTGGGAAGTCATGGTGC \\
COL-1 & F: CTGACGCATGGCCAAGAAG \\
OCN & R: CGTGCCATTGTGGCAGATA \\
& F: GGCGCTACCTCAACAATGG \\
& R: ATAGATGCGCTTGTAGGCG \\
\hline
\end{tabular}

F, forward; R, reverse; CKIP-1, casein kinase-2 interaction protein-1; Runx2, RUNX family transcription factor 2; ALP, alkaline phosphatase; COL-1, colicinogenic factor 1 ; OCN, bone $\gamma$-carboxyglutamate protein.

as the mean $\pm \mathrm{SD}$. All experiments were repeated $\geq 3$ times. An unpaired t-test was used for two group-comparison of the bone mass changes in CKIP-1 KO mice. A one-way ANOVA with Bonferroni correction $(\alpha=0.05)$ was used to analyze the data for each CD marker. A two-way ANOVA with Bonferroni correction $(\alpha=0.05)$ was used to analyze other data. $\mathrm{P}<0.05$ was considered to indicate a statistically significant difference.

\section{Results}

Bone formation can be regulated by CKIP-1. CKIP-1 KO and WT mice were used to investigate the relationship between CKIP-1 and bone formation. Following genotype identification (Fig. 1A), CKIP-1 ${ }^{-/}$mice were sacrificed and femoral bones were isolated for subsequent experiments. The 3D reconstruction of the micro-CT results identified increased compact cancellous bone in the femoral bones obtained from the KO group. Moreover, the BV/TV, Tb.N and Tb.Th in the KO group were increased compared with the WT group, and the opposite trend was observed for BS/BV and Tb.Sp. However, 3D reconstruction of the cortical femoral bones and quantitative analysis found similar results between the KO and WT groups, regardless of the Ct. Ar, Ct.ld.Pm, Ct.Od.Pm, Ct.Th and Ct.BV (Fig. 1B), which indicated that the role of CKIP-1 during cortical bone formation was insignificant. Moreover, H\&E staining was performed to investigate the differences between WT and KO mice. Gross observation of the femoral bones was consistent with the 3D reconstruction images, and the results of histomorphometry also identified increased bone formation in the $\mathrm{KO}$ group, accompanied by increased $\mathrm{Tb}$.Ar, Tb.Wi and Tb.N, and decreased Tb.Sp in the KO group for cancellous bone. However, no significant difference of Ct.Th between the WT and KO groups was observed for cortical bone (Fig. 1C). 

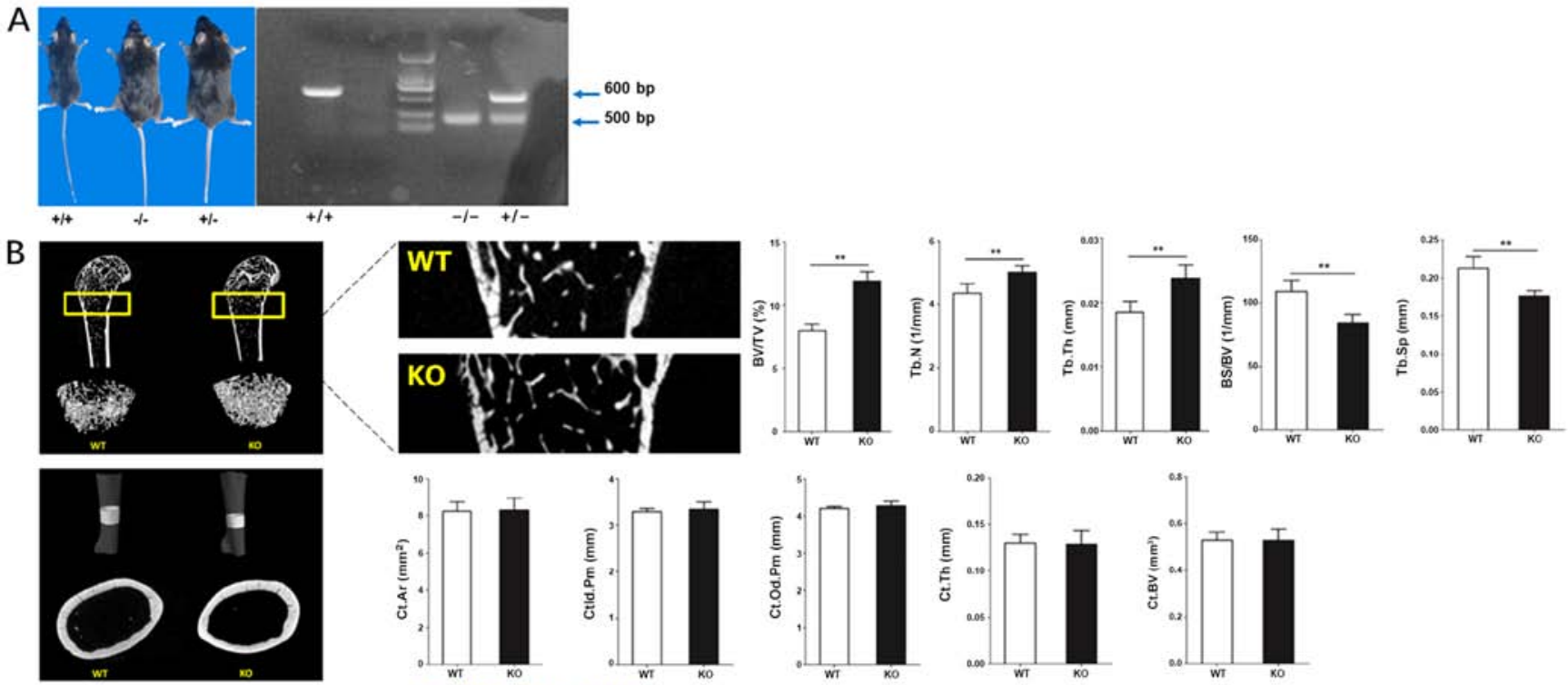

C

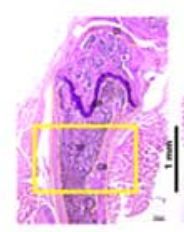

$40 \mathrm{X}$, WT

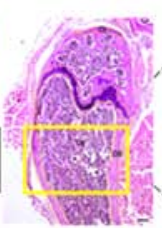

$40 \mathrm{X}, \mathrm{KO}$
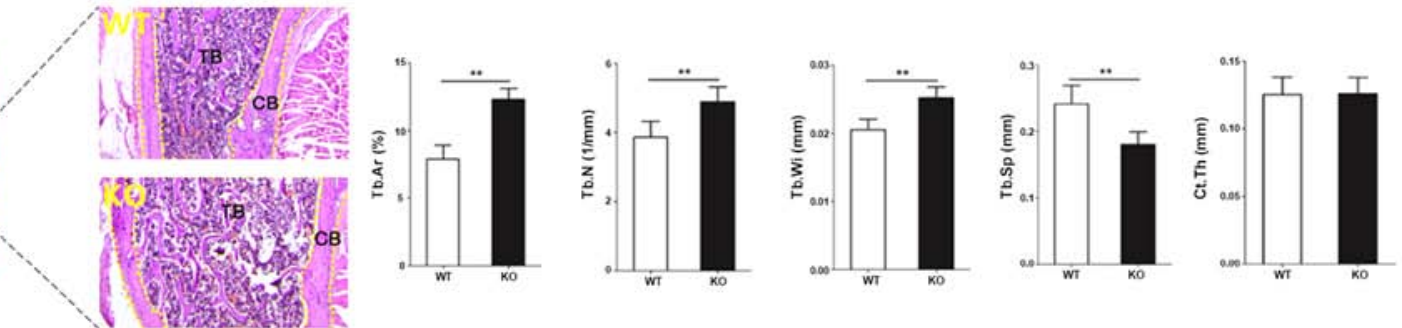

Figure 1. Bone formation can be negatively regulated by CKIP-1. (A) Gross observation and genotype identification of CKIP-1 knockout mice (CKIP-1 ${ }^{-1}$; $500 \mathrm{bp}$ ) using PCR. (B) Three-dimensional reconstruction of the cancellous and cortical bones of WT and CKIP-1 KO mice by micro-computed tomography. (C) H\&E staining and histological analysis were performed to evaluate the mass of cancellous and cortical bones of WT and KO mice. Magnification, $\mathrm{x} 40$. Rectangular boxes and the magnified images (magnification at x200 for micro-computed tomography and x120 for H\&E staining) display the regions of interests for quantitative analysis. ${ }^{* *} \mathrm{P}<0.01$ vs. WT group. CKIP-1, casein kinase-2 interaction protein-1; WT, wild-type; KO, knockout; TB, trabecular bone; CB, cortical bone; GP, growth plate; CI, cartilage; Tb.N, trabecular number; Tb.Sp, trabecular separation; Tb.Ar, trabecular area; BV/TV, bone volume/total volume ratio; Tb.Th, trabecular thickness; BS/BV, bone surface/bone volume; $\mathrm{Ct}$. Ar, cortical area; Ct.ld.Pm, cortical inner diameter perimeter; Ct.Od. Pm, cortical outer diameter perimeter; Ct.Th, cortical thickness; Ct.BV, cortical bone volume; Tb.Wi, trabecular width; H\&E, hematoxylin and eosin.

Identification of BMMSCs and OMSCs. Microstructures on bone surfaces before and after collagenase II digestion were observed by FE-SEM. It was demonstrated that the digested bone had a number of micro-holes on the surface (diameter, 70-80 microns), which may have allowed the stem cells to move across the bone surface (Fig. 2A). The collagenase-digested bone pieces were inoculated for conventional culture. On day 3 , stem cells were observed on the surface of the bone pieces, and the cells in the $\mathrm{KO}$ group displayed higher proliferation in generation passage 1 (P1) and P3 (Fig. 3B). In addition, it was determined that a small number of hematopoietic cells were present in the P1 generation, and stem cells were purified when passaged to the P3 generation (Fig. 2B). To identify BMMSCs and OMSCs, in vitro differentiation and detection of surface antigens using FCM were performed. Alizarin Red S staining on day 21 and Oil Red O staining results on day 14 suggested that digestion-derived MSCs had differentiated to osteogenic and adipogenic lineages, further indicating the multidirectional differentiation ability of MSCs (Fig. 2C). Furthermore, the FCM results identified high expression levels of CD29, CD44 and CD90 on the surface of BMMSCs and OMSCs, which were significantly higher compared with the expression level of CD31 and CD34, thus suggesting the presence of endothelial and hematopoietic cells.
Moreover, the expression levels of the aforementioned markers were not significantly different between the four groups, which suggested that neither CKIP-1 KO or the source of the cells altered surface marker expression (Fig. 2D).

Cell morphology, proliferation and apoptosis. After cell culture for 1 day, the morphology of BMMSCs and OMSCs was observed by FE-SEM. The results indicated that an increased number of MSCs were observed in the KO group at low magnification compared with the WT group. Furthermore, at high magnification, all cells displayed a spindle-like morphology; however, OMSCs were relatively large and fully spread, with thicker and higher levels of interlinked lamellipodia compared with BMMSCs (Fig. 3A). An MTT assay was used to assess cell proliferation in the various groups, and it was determined that the proliferation of MSCs in the $\mathrm{KO}$ group was significantly increased between days 3-5, and the proliferation rate of OMSCs in the WT and KO groups was increased compared with the BMMSC group (Fig. 3B). The results also indicated that the increased rate of proliferation of OMSCs was higher compared with BMMSCs, following CKIP-1 KO. Moreover, a clone formation assay was also performed on MSCs following a 2-week incubation. Consistent with the results of the MTT assay, cloning efficiency was 
A

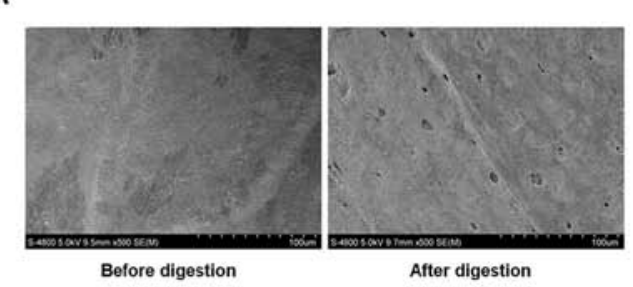

B

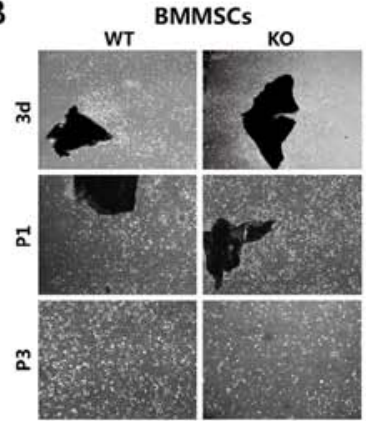

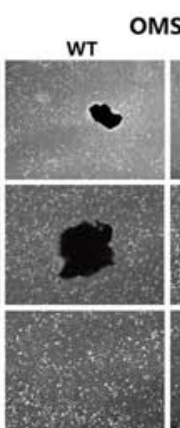
ко

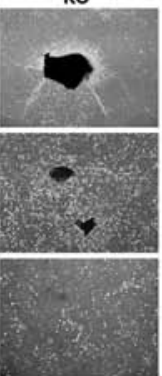

D
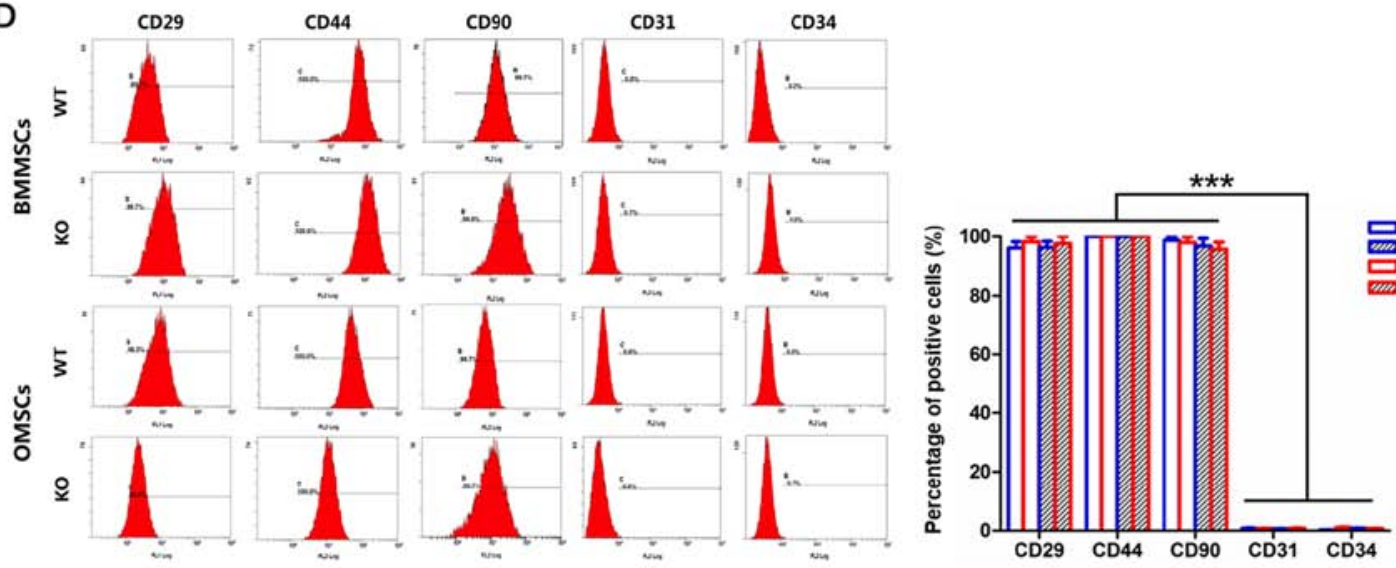

Figure 2. Isolation and identification of BMMSCs and OMSCs. (A) Observation of bone surfaces before and after collagenase II digestion using field emission scanning electron microscopy. (B) Primary culture and purification of MSCs by cell passaging. (C) Osteogenic and adipogenic differentiation of MSCs at 14 and 21 days of induction (magnification, $\mathrm{x} 40$ ). (D) Detection and quantitative analysis of the surface markers of MSCs using flow cytometry. ${ }^{* * *} \mathrm{P}<0.001$ vs. CD31 and CD34. BMMSCs, bone marrow-derived MSCs; OMSCs, orofacial bone-derived MSCs; MSCs, mesenchymal stem cells WT, wild-type; KO, knockout.

significantly increased in the KO group compared with the WT group, and the increased levels of OMSCs were more significant compared with BMMSCs (Fig. 3C). In addition, cell apoptosis was assessed using FCM and the results indicated that there was no significant difference in the rate of cell apoptosis between OMSCs (4.1\%) and BMMSCs $(3.8 \%)$ following CKIP-1 KO (Fig. 3D).

In vitro osteogenic differentiation. After 7 days of osteogenic induction, ALP activity was assessed in OMSCs and BMMSCs derived from $\mathrm{WT}$ and $\mathrm{KO}$ mice. The results indicated that osteogenic differentiation occurred in all cells, and ALP staining of OMSCs was highest in the KO group, but lowest in BMMSCs derived from WT mice. Moreover, quantitative analysis of ALP staining revealed that the ALP activity of MSCs in the $\mathrm{KO}$ group was increased compared with the WT group, and that ALP activity of OMSCs following CKIP-1 KO was increased compared with BMMSCs (Fig. 4A). Alizarin Red S staining was performed to assess osteogenic mineralization in the various groups following a 21-day incubation. The results indicated that the osteogenic ability in the KO group was increased compared with the WT group, and that the increase in osteogenesis of OMSCs was significantly higher compared with BMMSCs (Fig. 4B). In addition, to further investigate the aforementioned results, RT-qPCR was performed to detect the expression levels of CKIP-1 and osteogenesis-related genes (Runx2, ALP, COL-1 and OCN). It was demonstrated that CKIP-1 was not expressed in OMSCs and BMMSCs derived from CKIP-1 ${ }^{-/}$mice. However, following osteogenic induction for 7 days, the expression levels of Runx 2 and ALP were significantly increased in MSCs of the KO group compared with the WT group, and the increase in OMSCs was higher in the KO group compared with that of BMMSCs. Furthermore, the expression levels of COL-1 and OCN in MSCs following a 21-day incubation exhibited a similar trend (Fig. 4C).

In vivo ectopic bone formation. In order to further assess the difference in osteogenic differentiation ability between OMSCs and BMMSCs derived from WT and KO mice, MSCs were inoculated onto HA/ $\beta$-TCP scaffolds that were subsequently subcutaneously transplanted into nude mice at specific sites (Fig. 5A). The gross observation, H\&E staining and Masson-trichrome staining results of the samples 2 months post-transplantation are presented in Fig. 5B. The results revealed that the implants in the four groups survived and formed fibrous connective tissues. However, the amount of tissue formation differed, with OMSCs in the KO group exhibiting the highest levels of tissue formation and BMMSCs in the WT group having the lowest levels. The results of H\&E and Masson-trichrome staining indicated that a large number of collagen tissues and a certain amount of bone were formed in the four groups. Furthermore, quantitative analysis of $\mathrm{H} \& \mathrm{E}$ and Masson-trichrome staining demonstrated an increased area percentage of new bone in the $\mathrm{KO}$ group compared with the WT group, and also in the OMSC group compared with BMMSCs group. Consistent with the in vitro results, it was determined that ectopic new bone formation was significantly increased following CKIP-1 KO, and that OMSCs had an 
A

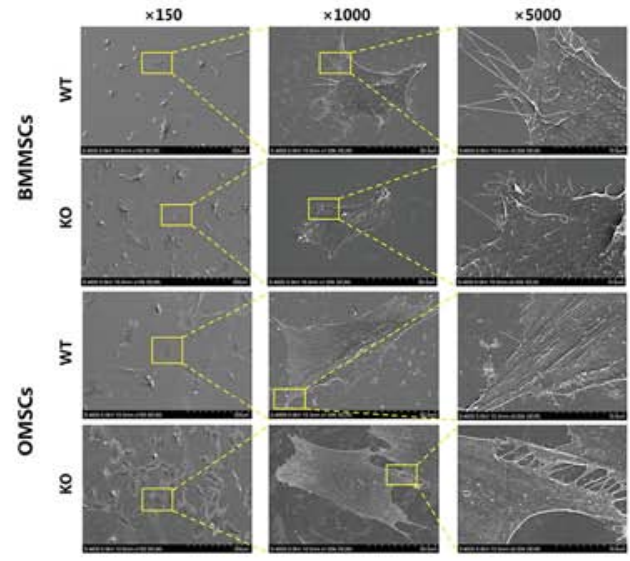

C

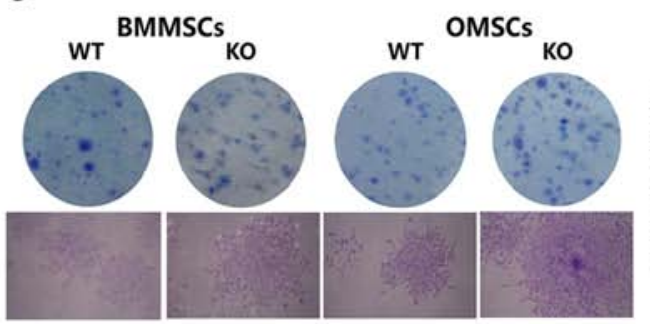

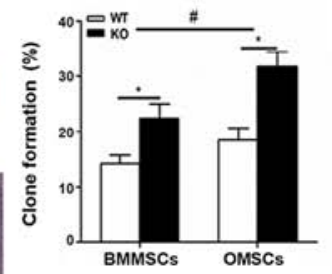

B
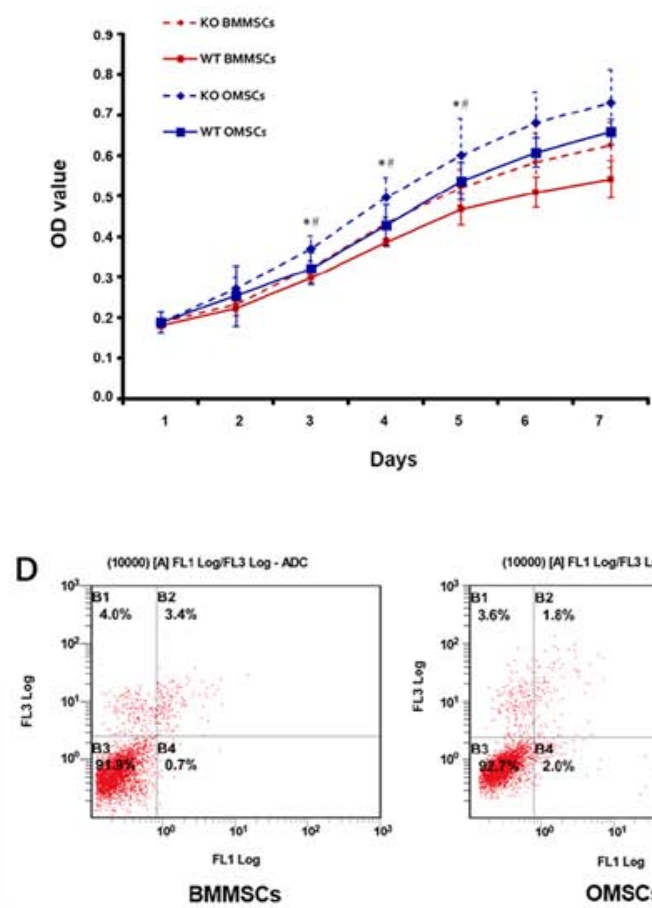

Figure 3. Morphology, proliferation and apoptosis of BMMSCs and OMSCs. (A) Observation of the morphology of BMMSCs and OMSCs in the WT and KO groups using field emission scanning electron microscopy. Proliferation of BMMSCs and OMSCs in the WT and KO groups assessed by (B) MTT and (C) clone formation assays (magnification, $\mathrm{x} 100$ ). (D) Apoptosis of BMMSCs and OMSCs in the KO group detected using flow cytometry. "P<0.05 vs. WT group; ${ }^{\#} \mathrm{P}<0.05$ vs. BMMSCs. BMMSCs, bone marrow-derived MSCs; OMSCs, orofacial bone-derived MSCs; MSCs, mesenchymal stem cells; WT, wild-type; $\mathrm{KO}$, knockout; OD, optical density.

A

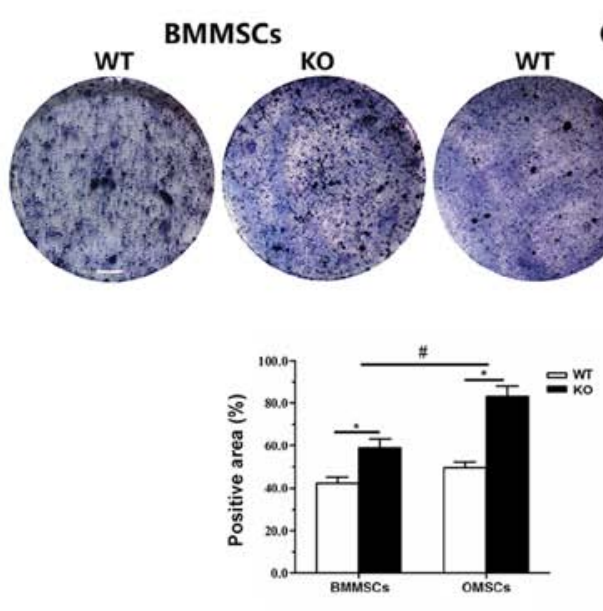

B
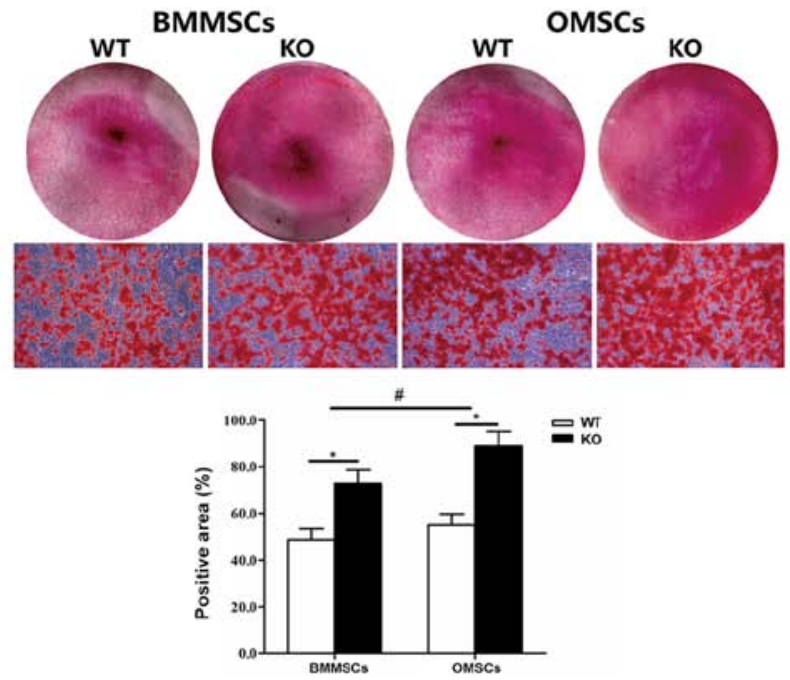

C

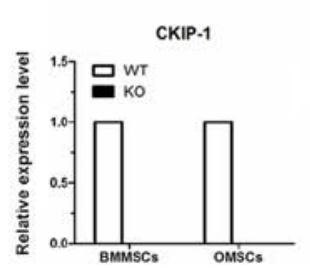

OMSCS
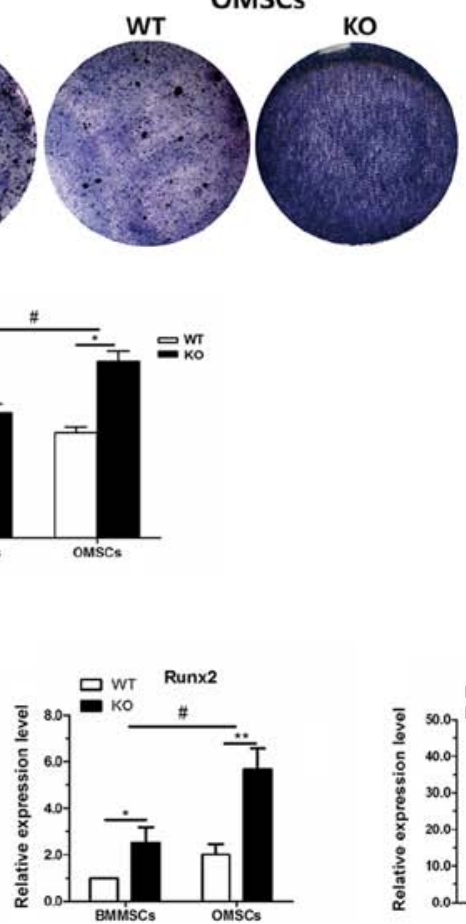
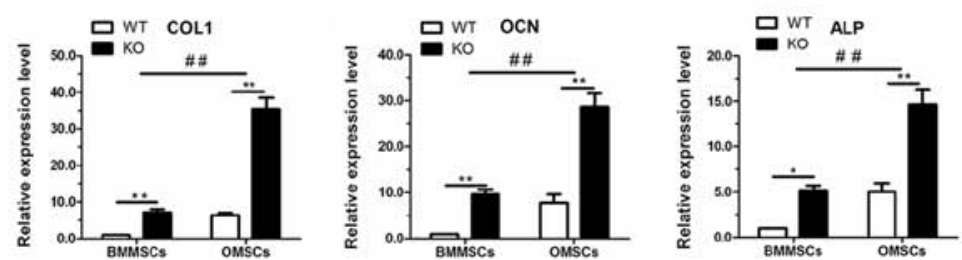

Figure 4. In vitro osteogenic differentiation ability of BMMSCs and OMSCs. (A) ALP staining and quantification of BMMSCs and OMSCs in the WT and KO groups after 7-day culture (magnification, x40). (B) Alizarin Red S staining and quantification of BMMSCs and OMSCs in the WT and KO groups after 21-day culture (magnification, $\mathrm{x} 40$ ). (C) Evaluation of the expression levels of CKIP-1 and the osteogenesis-related genes Runx2 and ALP after 7-day culture, and COL-1 and OCN after 21 -day culture. ${ }^{*} \mathrm{P}<0.05,{ }^{* *} \mathrm{P}<0.01$ vs. WT group; ${ }^{\#} \mathrm{P}<0.05,{ }^{\# *} \mathrm{P}<0.01$ vs. BMMSCs. BMMSCs, bone marrow-derived MSCs; OMSCs, orofacial bone-derived MSCs; MSCs, mesenchymal stem cells; WT, wild-type; KO, knockout; Runx2, RUNX family transcription factor 2; ALP, alkaline phosphatase; COL-1, colicinogenic factor 1; OCN, bone $\gamma$-carboxyglutamate protein. 
A

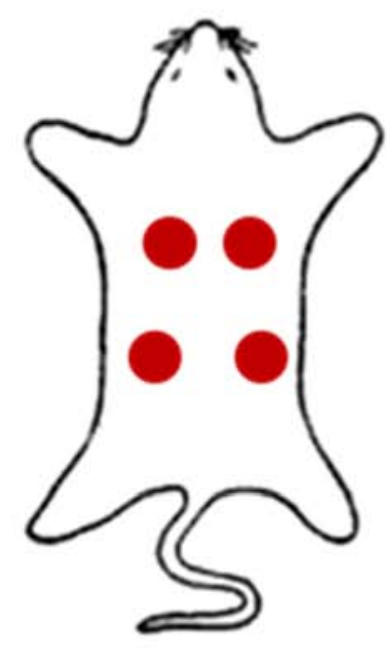

B
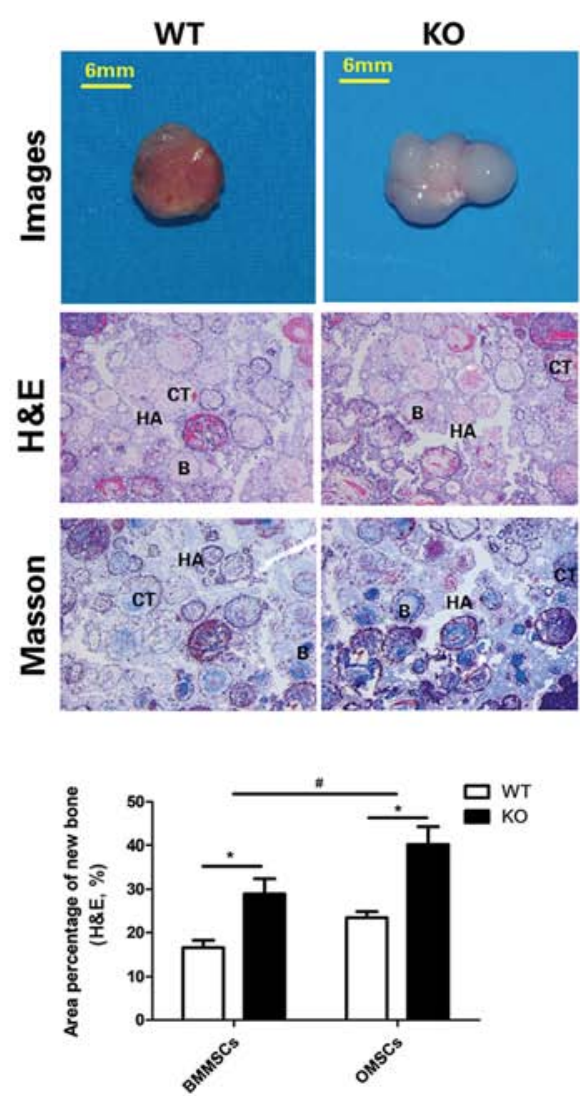

BMMSCs

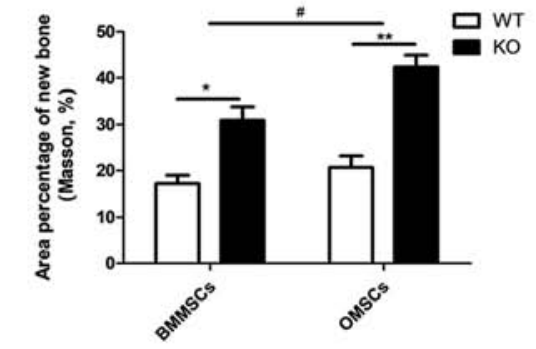

Figure 5. Gross observation and evaluation of ectopic bone formation. (A) Specific sites of the subcutaneous transplantation of the MSCs $+\mathrm{HA} / \beta-\mathrm{TCP}$ complex in nude mice. (B) Gross observation, H\&E and Masson-trichrome staining at 2 months post-subcutaneous transplantation of the complex in nude mice. Scale bar, $6 \mathrm{~mm}$; magnification, $\mathrm{x} 40 .{ }^{*} \mathrm{P}<0.05,{ }^{* *} \mathrm{P}<0.01$ vs. WT group; ${ }^{*} \mathrm{P}<0.05$ vs. BMMSCs. MSCs, mesenchymal stem cells; HA/ $\beta$-TCP, hydroxyapatite/tricalcium phosphate; WT, wild-type; BMMSCs, bone marrow-derived MSCs; CT, collagen tissues; B, bones; HA, HA/ $\beta-T C P$; H\&E, hematoxylin and eosin.

improved osteogenesis ability compared with BMMSCs in the KO groups.

\section{Discussion}

MSCs are a promising cell source for bone tissue engineering with multidirectional differentiation potential (4), and BMMSCs derived from the bone marrow of femoral bones and the ilium are commonly used (4). However, the mandible has a number of disadvantages, including anatomical limitations and a difficulty to isolate MSCs (20); therefore, few studies have focused on OMSCs. It has been reported that cortical bone is a novel and reliable source of MSCs, and that the collagenase digestion method is optimal for the isolation of MSCs (19-21). To the best of our knowledge, the extraction of MSCs from the mandible using the collagenase digestion method has not been previously reported; however, the potential effects of different isolation methods on the biological characteristics of MSCs may be avoided by using this method. In the present study, BMMSCs and OMSCs were successfully isolated and cultured using the collagenase digestion method.

The present results indicated that BMMSCs and OMSCs expressed the same cell surface markers with high expression levels of CD29, CD44 and CD90, which implied that MSCs can be successfully isolated and cultured from the mandible using the collagenase digestion method $(19,20,25)$. Furthermore, the isolated MSCs exhibited high purity, which provided further support for the use of the collagenase digestion method in future studies. In addition to the identification of MSCs, the FCM results also revealed no significant difference in the expression levels of cell surface markers between MSCs derived from CKIP-1 KO mice and WT mice, or between BMMSCs and OMSCs. Based on the markers that were investigated in the present study, the expression of cell surface markers could not be used to distinguish the two cell types, as expression was identical. However, whether BMMSCs and OMSCs share the same cell surface markers requires further investigation. It has been hypothesized that if cells display different cell surface markers, it suggests that the tissue origin has an influence on the expression of cell surface markers by MSCs, at least for the mandible and femoral bones.

The 3D reconstruction results of micro-CT and $\mathrm{H} \& \mathrm{E}$ staining indicated that the bone mass increased significantly following CKIP-1 KO, which was consistent with our previous study (18) and other studies $(17,26,27)$. Moreover, the present results further indicated that the main site of action of CKIP-1 is the cancellous bone, rather than the cortical bone. In addition, compared with MSCs in the WT group, the density of MSCs in the KO group was significantly increased, and differences between BMMSCs and OMSCs were also observed. It was demonstrated that the rate of apoptosis was slightly altered following CKIP-1 KO, which was opposite to a previous report that suggested CKIP-1 activates caspase-3 to promote 
apoptosis (28). Therefore, it was hypothesized that CKIP-1 may not affect the survival and apoptosis of MSCs in the absence of other complex stimuli. Furthermore, MSCs may have different mechanisms for the induction and inhibition of apoptosis compared with the cells assessed in a previous study (28). The present SEM results demonstrated that OMSCs had larger pseudopodia and an increased extension area compared with BMMSCs, which suggested that OMSCs were more conducive to scaffold adhesion. It has also been previously reported that OMSCs display an increased proliferation ability compared with BMMSCs $(29,30)$, which was consistent with the results obtained in the present study.

The present results indicated that the osteogenic differentiation ability of MSCs displayed a similar trend (29). A possible reason is that the embryonic origin of the two cell types is different; OMSCs are derived from neural crest cells in the neuroectoderm, while BMMSCs are derived from mesenchymal cells in the mesoderm (16). Therefore, ectoderm-derived MSCs may be more sensitive to CKIP-1, thus resulting in increased alterations to the proliferation and osteogenic differentiation ability of OMSCs following CKIP-1 KO. Furthermore, the methods of bone formation are different between the two types of MSC; ectodermal MSCs differentiate and develop into craniofacial bone via a unique mechanism of intramembranous ossification, while mesodermal MSCs form trunk and limb bones via endochondral ossification $(31,32)$. Moreover, CKIP-1 can negatively regulate bone formation by suppressing Smurf1-mediated degradation of Smad (17). Based on the ossification mechanisms and CKIP-1, it was hypothesized that CKIP-1 may have an important role in the osteogenesis of osteoblasts compared with osteogenesis by chondrogenic differentiation, and that BMP signaling may be involved. In addition, CKIP-1 may be differentially expressed and exhibit different intensities of action in various cell types. Furthermore, it was speculated that the lower expression of CKIP-1 in OMSCs may induce increased proliferation and improved osteogenic differentiation ability (17). It has also been revealed that OMSCs have a stronger osteogenic differentiation ability compared with BMMSCs, which may be related to homeobox A2 (Hoxa2) gene expression, which is primarily expressed in femoral trunk bones and not in orofacial bones (33). Moreover, Hoxa 2 can inhibit the expression of osteogenesis-related molecules that are associated with intramembrane ossification in BMMSCs, thus resulting in a decrease in the biological characteristics of BMMSCs (33). Therefore, a possible explanation for the differential effects of CKIP-1 in OMSCs and BMMSCs may be that the corresponding signaling pathways are different.

To further investigate whether the differences of osteogenic capacity identified in vitro were also present in vivo, an ectopic osteogenesis experiment was performed in the present study, which involved the formation of bone tissue in typically boneless areas (34). Calcium phosphates are biocompatible ceramic compounds that are involved in the formation of bones (35-37). Moreover, the combination of HA and $\beta$-TCP can induce new bone formation at non-osseous sites (35-37), and therefore, $\mathrm{HA} / \beta$-TCP was selected as the scaffold material for the ectopic osteogenesis experiment. Consistent with the in vitro results, $\mathrm{H} \& \mathrm{E}$ and Masson-trichrome staining results further suggested that the osteogenic differentiation ability of MSCs was enhanced following CKIP-1 KO, and that the osteogenic differentiation ability of OMSCs was increased compared with BMMSCs.

In conclusion, the effects of CKIP-1 on the proliferation and osteogenesis of MSCs derived from the mandible and femoral bones were investigated using CKIP- $1^{-/-}$mice. The present results indicated the site specificity of MSC proliferation and osteogenesis, and revealed that OMSCs exhibited an enhanced sensitivity to CKIP-1. Therefore, the negative regulation of CKIP-1 in OMSCs may be a promising strategy for the repair of maxillofacial bone defects.

\section{Acknowledgements}

The authors would like to thank Professor Zhang Lingqiang (Institute of Radiation Medicine, Academy of Military Medical Sciences) for the kind donation of Ckip-1 ${ }^{-/-}$C57BL/6J mice.

\section{Funding}

The present study was supported by the National Natural Science Foundation of China (grant no. 81670803)

\section{Availability of data and materials}

All data generated or analyzed during this study are included in this published article.

\section{Authors' contributions}

$\mathrm{XH}, \mathrm{YS}$ and LK conceptualized the study. $\mathrm{XH}$ and WS designed the method. Software was provided by BC. BC and LW performed data analysis. YZ provided resources and interpreted the data. Data acquisition was performed by $\mathrm{YH}$. XH wrote the original draft preparation, and $\mathrm{XH}$ and WS reviewed and edited the manuscript. YS supervised the study. Project administration and funding acquisition was the responsibility of LK. All authors read and approved the final manuscript.

\section{Ethics approval and consent to participate}

All experimental procedures adhered to the principles stated in the Guide for the Care and Use of Laboratory Animals (updated 2011; National Institutes of Health), and were approved by the Experimental Animal Usage and Welfare Ethics Committee of the Fourth Military Medical University.

\section{Patient consent for publication}

Not applicable.

\section{Competing interests}

The authors declare that they have no competing interests.

\section{References}

1. Cicciù M, Cervino G, Herford AS, Famà F, Bramanti E, Fiorillo L, Lauritano F, Sambataro S, Troiano G and Laino L: Facial bone reconstruction using both marine or non-marine bone substitutes: Evaluation of current outcomes in a systematic literature review. Mar Drugs 16: E27, 2018. 
2. El-Rashidy AA, Roether JA, Harhaus L, Kneser U and Boccaccini AR: Regenerating bone with bioactive glass scaffolds: A review of in vivo studies in bone defect models. Acta Biomater 62: 1-28, 2017.

3. Leyendecker A Jr, Pinheiro CG, Fernandes TL and Bueno DF: The use of human dental pulp stem cells for in vivo bone tissue engineering: A systematic review. J Tissue Eng: Jan 17, 2018 (Epub ahead of print). doi: 10.1177/2041731417752766

4. Buduru SD, Gulei D, Zimta AA, Tigu AB, Cenariu D and Berindan-Neagoe I: The potential of different origin stem cells in modulating oral bone regeneration processes. Cells 8: E29, 2019.

5. He S, Yang S, Zhang Y, Li X, Gao D, Zhong Y, Cao L, Ma H, Liu Y, Li G, et al: LncRNA ODIR1 inhibits osteogenic differentiation of hUC-MSCs through the FBXO25/H2BK120ub/ H3K4me3/OSX axis. Cell Death Dis 10: 947, 2019.

6. Carthew J, Donderwinkel I, Shrestha S, Truong VX, Forsythe JS and Frith JE: In situ miRNA delivery from a hydrogel promotes osteogenesis of encapsulated mesenchymal stromal cells. Acta Biomater 101: 249-261, 2020

7. Ouyang Z, Tan T, Zhang X, Wan J, Zhou Y, Jiang G, Yang D, Guo X and Liu T: CircRNA hsa_circ_0074834 promotes the osteogenesis-angiogenesis coupling process in bone mesenchymal stem cells (BMSCs) by acting as a ceRNA for miR-942-5p. Cell Death Dis 10: 932, 2019.

8. Zhou J and Zhao L: Multifunction Sr, Co and F co-doped microporous coating on titanium of antibacterial, angiogenic and osteogenic activities. Sci Rep 6: 29069, 2016.

9. Prasopthum A, Cooper M, Shakesheff KM and Yang J: Three-dimensional printed scaffolds with controlled micro-/nanoporous surface topography direct chondrogenic and osteogenic differentiation of mesenchymal stem cells. ACS Appl Mater Interfaces 11: 18896-18906, 2019.

10. Aghajani F, Hooshmand T, Khanmohammadi M, Khanjani S, Edalatkhah H, Zarnani A-H and Kazemnejad S: Comparative immunophenotypic characteristics, proliferative features, and osteogenic differentiation of stem cells isolated from human permanent and deciduous teeth with bone marrow. Mol Biotechnol 58: 415-427, 2016.

11. Isobe Y, Koyama N, Nakao K, Osawa K, Ikeno M, Yamanaka S, Okubo Y, Fujimura K and Bessho K: Comparison of human mesenchymal stem cells derived from bone marrow, synovial fluid, adult dental pulp, and exfoliated deciduous tooth pulp. Int J Oral Maxillofac Surg 45: 124-131, 2016.

12. Peng L, Jia Z, Yin X, Zhang X, Liu Y, Chen P, Ma K and Zhou C: Comparative analysis of mesenchymal stem cells from bone marrow, cartilage, and adipose tissue. Stem Cells Dev 17: 761-773, 2008.

13. Lotfy A, Salama M, Zahran F, Jones E, Badawy A and Sobh M: Characterization of mesenchymal stem cells derived from rat bone marrow and adipose tissue: A comparative study. Int J Stem Cells 7: 135-142, 2014.

14. Khalilifar MA, Baghaban Eslaminejad MR, Ghasemzadeh M, Hosseini $\mathrm{S}$ and Baharvand $\mathrm{H}$ : In vitro and in vivo comparison of different types of rabbit mesenchymal stem cells for cartilage repair. Cell J 21: 150-160, 2019.

15. Lloyd B, Tee BC, Headley C, Emam H, Mallery S and Sun Z: Similarities and differences between porcine mandibular and limb bone marrow mesenchymal stem cells. Arch Oral Biol 77: 1-11, 2017.

16. Chai $\mathrm{Y}$ and Maxson RE Jr: Recent advances in craniofacial morphogenesis. Dev Dyn 235: 2353-2375, 2006.

17. Lu K, Yin X, Weng T, Xi S, Li L, Xing G, Cheng X, Yang X, Zhang L and He F: Targeting WW domains linker of HECT-type ubiquitin ligase Smurf1 for activation by CKIP-1. Nat Cell Biol 10: 994-1002, 2008.

18. Zhou ZC, Che L, Kong L, Lei DL, Liu R and Yang XJ: CKIP-1 silencing promotes new bone formation in rat mandibular distraction osteogenesis. Oral Surg Oral Med Oral Pathol Oral Radiol 123: e1-e9, 2017.

19. Guo Z, Li H, Li X, Yu X, Wang H, Tang P and Mao N: In vitro characteristics and in vivo immunosuppressive activity of compact bone-derived murine mesenchymal progenitor cells Stem Cells 24: 992-1000, 2006.
20. Zhu H, Guo ZK, Jiang XX, Li H, Wang XY, Yao HY, Zhang Y and Mao N: A protocol for isolation and culture of mesenchymal stem cells from mouse compact bone. Nat Protoc 5: 550-560, 2010.

21. Short BJ, Brouard N and Simmons PJ: Prospective isolation of mesenchymal stem cells from mouse compact bone. Methods Mol Biol 482: 259-268, 2009.

22. Liang J, Xu S, Shen M, Cheng B, Li Y, Liu X, Qin D, Bellare A and Kong L: Osteogenic activity of titanium surfaces with hierarchical micro-/nano-structures obtained by hydrofluoric acid treatment. Int J Nanomedicine 12: 1317-1328, 2017.

23. Song W, Ma Z, Zhang Y and Yang C: Autophagy plays a dual role during intracellular siRNA delivery by lipoplex and polyplex nanoparticles. Acta Biomater 58: 196-204, 2017.

24. Livak KJ and Schmittgen TD: Analysis of relative gene expression data using real-time quantitative PCR and the 2(-Delta Delta C(T)) methods Methods 25: 402-408, 2001.

25. Dominici M, Le Blanc K, Mueller I, Slaper-Cortenbach I, Marini F, Krause D, Deans R, Keating A, Prockop DJ and Horwitz E: Minimal criteria for defining multipotent mesenchymal stromal cells. The International Society for Cellular Therapy position statement. Cytotherapy 8: 315-317, 2006.

26. Zhang L, Wu K, Song W, Xu H, An R, Zhao L, Liu B and Zhang Y: Chitosan/siCkip-1 biofunctionalized titanium implant for improved osseointegration in the osteoporotic condition. Sci Rep 5: 10860, 2015.

27. Zhang X, Wang Q, Wan Z, Li J, Liu L and Zhang X: CKIP-1 knockout offsets osteoporosis induced by simulated microgravity. Prog Biophys Mol Biol 122: 140-148, 2016

28. Zhang L, Xing G, Tie Y, Tang Y, Tian C, Li L, Sun L, Wei H, Zhu Y and He F: Role for the pleckstrin homology domain-containing protein CKIP-1 in AP-1 regulation and apoptosis. EMBO J 24: 766-778, 2005

29. Aghaloo TL, Chaichanasakul T, Bezouglaia O, Kang B, Franco R, Dry SM, Atti E and Tetradis S: Osteogenic potential of mandibular vs. long-bone marrow stromal cells. J Dent Res 89: 1293-1298, 2010.

30. Yamaza T, Ren G, Akiyama K, Chen C, Shi Y and Shi S: Mouse mandible contains distinctive mesenchymal stem cells. J Dent Res 90: 317-324, 2011.

31. Karaplis AC: Embryonic development of bone and regulation of intramembranous and endochondral bone formation. In: Principles of Bone Biology. Academic Press, Cambridge, MA, pp53-84, 2002.

32. Helms JA and Schneider RA: Cranial skeletal biology. Nature 423: 326-331, 2003

33. Leucht P, Kim JB, Amasha R, James AW, Girod S and Helms JA: Embryonic origin and Hox status determine progenitor cell fate during adult bone regeneration. Development 135: 2845-2854, 2008.

34. Scott MA, Levi B, Askarinam A, Nguyen A, Rackohn T, Ting K, Soo C and James AW: Brief review of models of ectopic bone formation. Stem Cells Dev 21: 655-667, 2012.

35. Gallinetti S, Canal C, Ginebra MP and Ferreira J: Development and characterization of biphasic hydroxyapatite/ $\beta$-TCP cements. J Am Ceram Soc 97: 1065-1073, 2014.

36. Valentim RMB, Andrade SMC, Dos Santos MEM, Santos A, Pereira V, dos Santos I, Dias C and dos Reis M: Composite based on biphasic calcium phosphate (HA/ $\beta-\mathrm{TCP})$ and nanocellulose from the açaí tegument. Materials (Basel) 11: E2213, 2018.

37. Ishack S, Mediero A, Wilder T, Ricci JL and Cronstein BN: Bone regeneration in critical bone defects using three-dimensionally printed $\beta$-tricalcium phosphate/hydroxyapatite scaffolds is enhanced by coating scaffolds with either dipyridamole or BMP-2. J Biomed Mater Res B Appl Biomater 105: 366-375, 2017.

This work is licensed under a Creative Commons Attribution-NonCommercial-NoDerivatives 4.0 International (CC BY-NC-ND 4.0) License. 